\title{
Study on the resistance mechanism via outer membrane protein OprD2 and metal $\beta$-lactamase expression in the cell wall of Pseudomonas aeruginosa
}

\author{
SHUANGQI CAI ${ }^{1}$, YIQIANG CHEN ${ }^{1}$, DEZHI SONG $^{2}$, JINLIANG KONG ${ }^{1}$, YANBIN WU $^{1}$ and HUASONG LU ${ }^{1}$ \\ ${ }^{1}$ Department of Respiratory Medicine, The First Affiliated Hospital of Guangxi Medical University; \\ ${ }^{2}$ Department of Microbiology, Guangxi Medical University, Nanning, Guangxi 530021, P.R. China
}

Received April 19, 2016; Accepted August 26, 2016

DOI: $10.3892 /$ etm.2016.3690

\begin{abstract}
The aim of the present study was to evaluate the imipenem-resistant mechanism via the outer membrane protein (OMP) OprD2 and metal $\beta$-lactamase expression in the cell wall of Pseudomonas aeruginosa. The Pseudomonas aeruginosa was clinically separated and validated by VITEK-2 full-automatic bacteria analyzer. Drug resistance, sensitive antibiotics and minimum inhibitory concentration (MIC) were tested using the drug sensitivity analysis system. The phenotype positive strains of MBL genes were screened using the Kirby-Bauer diffusion method by adding metal ion-chelating agent EDTA on the imipenem susceptibility paper. IMP-1, VIM-1 and SPM metaloenzyme genes were tested by polymerase chain reaction (PCR)-telomeric repeat amplification protocol (TRAP). The OMP OprD2 genes were tested by PCR-TRAP, and the protein expression was tested using western blot analysis. The location of OMP OprD2 was confirmed using the sodium salicylate inhibition test. The results showed that 80 portions $(40 \%)$ of MBL-positive strains were screened out of 200 specimens. Imipenem-resistant Pseudomonas aeruginosa (IRPA) and MIC values were significantly higher than quality control bacteria and control bacteria $(\mathrm{P}<0.05)$. A total of 35 cases with IMP-1 positive, 20 with VIM-1 positive, 16 with SPM positive, 5 with 2 positive genes and 4 with 3 positive genes were screened among MBL positive strains. A total of 150 portions $(75 \%)$ of OprD2 deficiencies were screened from 200 specimens. The standard strains and sensitive strains showed OprD2 protein bands at $45 \mathrm{kDa}$ while no OprD2 protein bands appeared in
\end{abstract}

Correspondence to: Dr Yiqiang Chen or Dr Jinliang Kong, Department of Respiratory Medicine, The First Affiliated Hospital of Guangxi Medical University, 6 Shuangyong Road, Nanning, Guangxi 530021, P.R. China

E-mail: chen_yiqiang1@163.com

E-mail: kj1071@126.com

Key words: Pseudomonas aeruginosa, outer membrane protein OprD2, metal $\beta$-lactamase expression, imipenem
OprD2 deficiency strains. It was in accordance with gene detection. In conclusion, OMP OprD2 deficiency and MBL phenotype positivity may be important mechanisms of IRPA.

\section{Introduction}

Pseudomonas aeruginosa is not only a common conditional pathogen but also one of major pathogenic bacteria that may cause outbreak of nosocomial infections. However, increasing imipenem-resistant Pseudomonas aeruginosa (IRPA) results in difficulties (1) in clinical treatment. Previous studies showed that metal MBL $\beta$-lactamase (MBL) could split the $\beta$-lactam antibiotic $\beta$-lactamase ring to make antibiotics ineffective on bacteria (2). It is one of the important drug resistance mechanisms for the bacteria. MBL-mediated IRPA was identified and confirmed and includes seven types - IMP, VIM, GIM, SPM, SIM, AIM and NDM-1, of which IMP and VIM have the closest clinical relationships (3).

The micropore proteins OprC, OprD and OprE are major parts of the outer membrane protein (OMP) of Pseudomonas aeruginosa. They are also specialized pathways for small molecule hydrophilic substances to enter into the bacteria. OprD2 protein is a specific pathway for imipenem entering into the Pseudomonas aeruginosa. It has ligand specificity with loci-specific binding of the imipenem. It has no cross resistance (4) with other $\beta$-lactam antibiotics. Previous findings have shown a decrease in OMP OprD2 expression and deficiency is a main cause for Pseudomonas aeruginosa having resistance to carbapenems, leading to a decrease in OMP permeability, resulting in Pseudomonas aeruginosa having resistance to carbapenems in the end (5).

The present study aimed to investigate the mechanism of IRPA from the angle of MBL generation and OMP OprD2 deficiency. It has great relevance to clinically reasonable and effective use of antibiotics.

\section{Materials and methods}

Bacterial isolation and identification. A total of 200 specimens derived from each clinical department of The First Affiliated Hospital of Guangxi Medical University (Guangxi, China) from January, 2015 to January, 2016 were selected and 
the Pseudomonas aeruginosa standard strain ATCC27853 was taken as a quality control strain while the IRPA acted as the control. They were validated by the VITEK-2 full-auto bacteria identification/drug susceptibility analysis system (Biomerieux, Marcy l'Étoile, France), as well as the drug sensitivity test. Based on 2012 standard constituted by American Clinical and Laboratory Standard Association as references, the minimum inhibitory concentration (MIC) values of IRPA were collected after being tested by agar plate double dilution method.

MBL phenotype screened by Kirby-Bauer diffusion method. Experimental strains were produced to $0.5 \mathrm{M}$ bacterial suspension by using sterilized saline water and were evenly coated on a Müller-Hinton (M-H) plate in length of $9 \mathrm{~cm}$. Two $10 \mu \mathrm{g}$ IMP drug sensitivity test papers (Merck Millipore, Billerica, MA, USA) were pasted of which one with $10 \mu$ l EDTA in concentration of $500 \mathrm{mmol} / \mathrm{l}$. The $\mathrm{M}-\mathrm{H}$ plate with pasted drug sensitivity test papers was placed into a constant temperature incubation under $35^{\circ} \mathrm{C}$ for $18-24 \mathrm{~h}$. Subsequently, the diameter of inhibition zone of IMP drug sensitivity test papers with EDTA and only with IMP was tested. The diameter of inhibition zone of IMP papers with EDTA was $\geq 5 \mathrm{~mm}$ than only with IMP paper, which was the MBL phenotype -positive strain.

Polymerase chain reaction (PCR) testing metalloenzyme genes IMP-1, VIM-1, SPM and OprD2. PCR Premix Taq regent and primer synthesis (Takara Bio, Dalian, China), electrophoresis and DNA marker (Beijing TransGen Biotech Co., Ltd., Beijing, China), and PCR (Bio-Rad, Berkeley, CA, USA) were used in this study. To extract total DNA, a single colony was taken from blood agar and placed in $500 \mu \mathrm{l}$ of tri-distilled water at $100^{\circ} \mathrm{C}$ for $10 \mathrm{~min}$, in a refrigerated air dryer under $4^{\circ} \mathrm{C}$ at $8,000 \mathrm{x}$ g for $10 \mathrm{~min}$. The bacterial DNA in supernatant $(100 \mu \mathrm{l})$ was drawn and removed into another sterile centrifuge tube and stored at $-20^{\circ} \mathrm{C}$ for standby.

The reaction mixture consists of $2 \mathrm{X}$ Taq MasterMix $(25 \mu \mathrm{l})$, upstream and downstream primers $(10 \mu \mathrm{M})$ for each $2 \mu \mathrm{l}$, template DNA $(4 \mu \mathrm{l}), \mathrm{ddH}_{2} \mathrm{O}(17 \mu \mathrm{l})$ to make up final volume of $50 \mu 1$. The PCR conditions for IMP-1, VIM-1 and SPMs were pre-denaturation at $94^{\circ} \mathrm{C}$ for $4 \mathrm{~min}$, denaturation at $94^{\circ} \mathrm{C}$ for $30 \mathrm{sec}$, annealing at $56^{\circ} \mathrm{C}$ for $30 \mathrm{sec}$ and extension at $72^{\circ} \mathrm{C}$ for $1 \mathrm{~min}$ for 35 cycles and final extension at $72^{\circ} \mathrm{C}$ for $10 \mathrm{~min}$. The PCR conditions for OprD2 PC were pre-denaturation at $94^{\circ} \mathrm{C}$ for $5 \mathrm{~min}$, denaturation at $94^{\circ} \mathrm{C}$ for $30 \mathrm{sec}$, annealing at $55^{\circ} \mathrm{C}$ for $30 \mathrm{sec}$ and extension at $72^{\circ} \mathrm{C}$ for $1 \mathrm{~min}$ for total of 35 cycles and re-extension at $72^{\circ} \mathrm{C}$ for $10 \mathrm{~min}$. PCR products were separated by gel electrophoresis (AGE), $5 \mu 1$ of PCR products was electrophoresed with a voltage of $110 \mathrm{~V}$ for $40 \mathrm{~min}$. The gel image was captured with UV light, and the sequences were logged in GenBank for comparison and analysis. The primers used are listed in Table I.

Western blotting for OprD2 protein expression. Total protein was extracted based on the specifications of bacterial membrane protein extraction kits and the protein concentration was tested using the BCA protein titer kits. It was prepared to $5.0 \mathrm{mg} / \mathrm{ml}$ and stored at $-80^{\circ} \mathrm{C}$. OMP was separated by SDS-PAGE electrophoresis method (Beijing Liuyi Instrument Factory, Beijing, China) for staining and decolorizing using Coomassie Brilliant
Table I. Primer sequences used in this study.

Band

Gene Primer sequence length, bp

IMP-1 F: 5'-CTACCGCAGCAGAGTCTTTG-3'

R: 5'-AACCAGTTTTGCCTTACCAT-3

VIM-1 F: 5'-AGTGGTGAGTATCCGACAG-3'

R: 5'-ATGAAAGTGCGTGGAGAC-3'

SPM F: 5'-GCGTTTTGTTTGTTGCTC-3'

R: 5'- TTGGGGATGTGAGACTAC-3'

OprD2 F: 5'-GCGCATCTCCAAGACCATG-3' R: 5'-GCCACGCGATTTGACGGAG-3'

Blue fast staining liquid after electrophoresis. The results were analyzed by gel-imaging system (Syngene, Frederick, MD, USA).

OprD2 position confirmed by sodium salicylate inhibition test. The imipenem-sensitive Pseudomonas aeruginosa was respectively inoculated in $2 \mathrm{M}-\mathrm{H}$ plates, 1 with $32 \mathrm{mmol} / \mathrm{l}$ of sodium salicylate and another without sodium salicylate. The imipenem drug susceptibility discs were, respectively, pasted in two M-H plates, to incubate at $37^{\circ} \mathrm{C}$ overnight to observe the size of IMP inhibition zone. The OMP was extracted from IMP-sensitive strains in the $\mathrm{MH}$ plate with or without sodium salicylate for SDS-PAGE for electrophoresis. The OMP D2 location was determined based on features of OMP D2 being suppressed by salicylic acid, namely the OMP D2 grown in the M-H plate with sodium salicylate was suppressed by salicylic acid and not expressed. During electrophoresis, the protein bands were missing around $45 \mathrm{kDa}$. The OMP D2 grown in the M-H plate without sodium salicylate was not suppressed and the protein bands turned up around $45 \mathrm{kDa}$ during electrophoresis.

Statistical analysis. Data analysis was carried out using the SPSS 19.0 software (SPSS, Inc., Chicago, IL, USA). Quantitative data were expressed as mean \pm standard deviation. The comparison among groups was carried out using ANOVA. The quantitative data were expressed by case number or percentage (\%). Comparison among groups was performed by $\chi^{2}$ test. $\mathrm{P}<0.05$ was considered to indicate a statistically significant difference.

\section{Results}

Comparisons of MIC value. A total of 80 portions (40\%) of MB-positive strains were screened from 200 specimens. MIC value of IRPA was significantly higher than quality control bacteria and control bacteria. The difference was of statistical significance $[(265.4 \pm 43.2)$ vs. $(0.9 \pm 0.2),(1.2 \pm 0.3) \mu \mathrm{g} / \mathrm{ml}$, $\mathrm{F}=152.342, \mathrm{P}<0.001]$, as shown in Fig. 1 .

$M B L$ gene test. A total of 35 cases with IMP-1 positivity were screened out from 80 portions of MBL positive strains, 20 with VIM-1 positive, 16 with SPM positive, 5 with 2 positive genes and 4 with 3 positive genes, as shown in Fig. 2. 


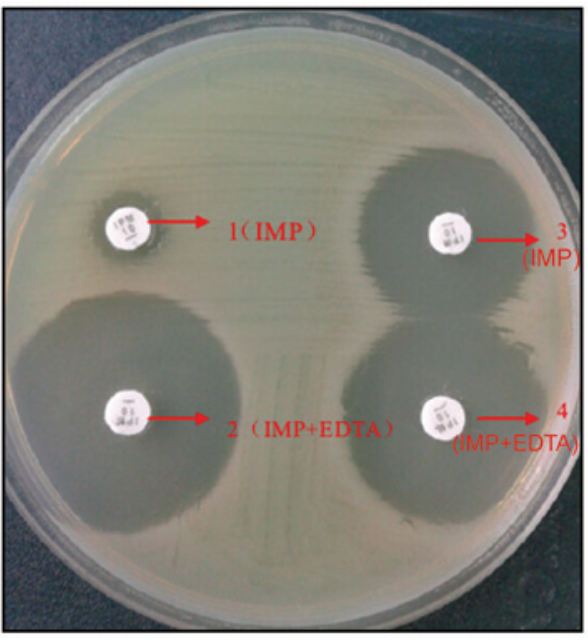

Figure 1. Screening of MBL phenotype with Kirby-Bauer disk diffusion method (1 and 2, drug-resistance bacteria inhibition zone; 3 and 4, sensitive bacteria inhibition zone; 2 , positive MBL phenotype; 4, negative MBL phenotype).

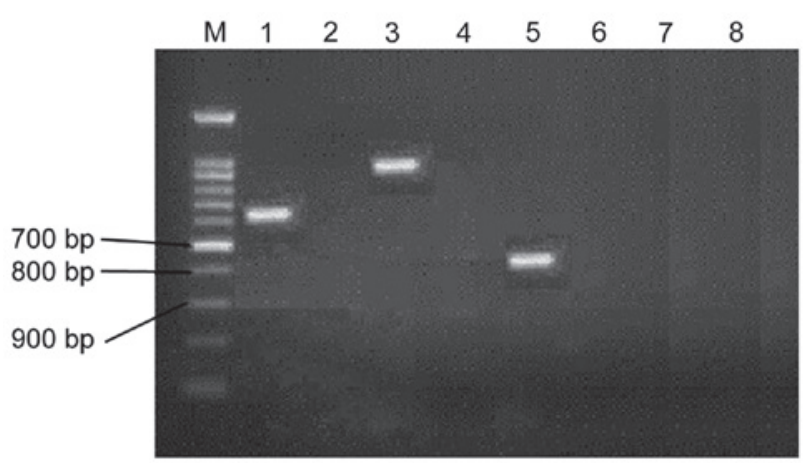

Figure 2. MBL gene electrophoretogram (1, positive IMP-1; 2, negative IMP-1; 3, positive VIM-1; 4, negative VIM-1; 5, positive SPM; 6, negative SPM; 7 , standard strains; 8 , sensitive bacteria).

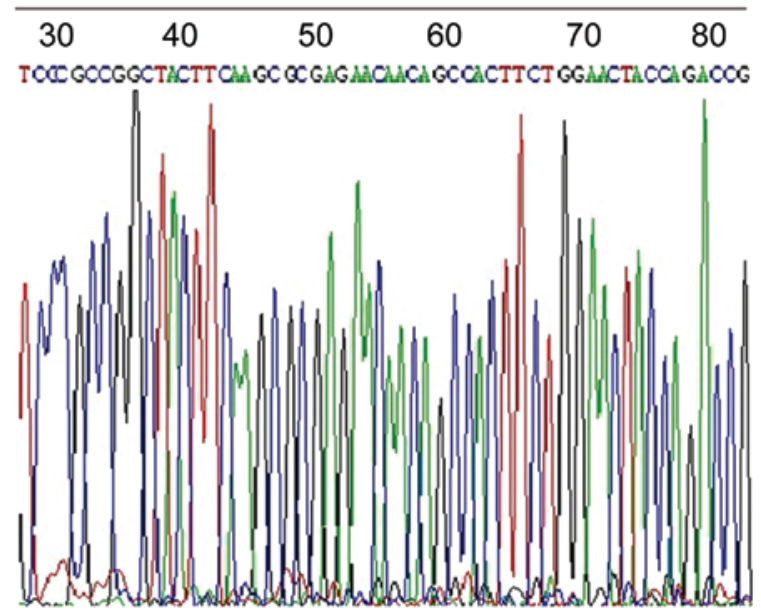

Figure 3. OprD2 gene sequencing diagram.

OprD2 gene and protein expression. A total of 150 portions (75\%) of OprD2 deficiencies were screened out of 200 specimens. Gene sequencing is shown in Fig. 3. The OprD2 protein bands appeared at $45 \mathrm{kDa}$ in the standard strains and sensitive

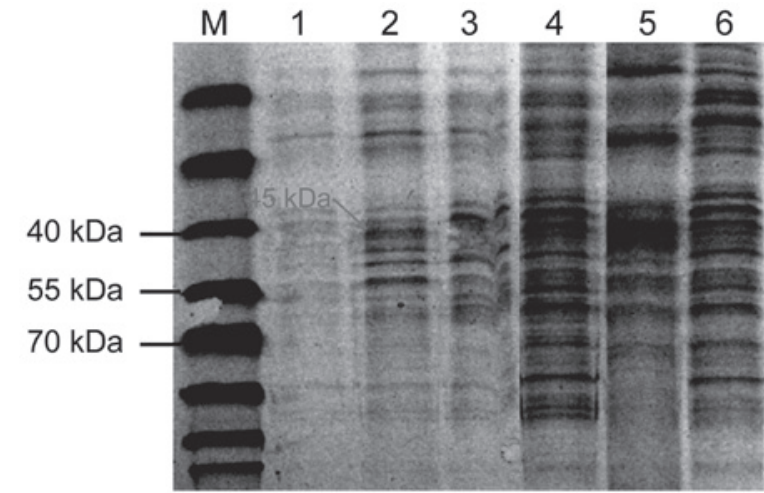

Figure 4. OprD2 protein electrophoretogram (1, sensitive strain containing sodium salicylate; 2 , sensitive strain without sodium salicylate growth; 3 , OprD2 missing drug resistant strains; 4, positive OprD2 drug-resistant strain; 5 , sensitive strain; 6 , standard strain).

strains, and no OprD2 protein bands appeared in OprD2 deficiency strains. The results were in accordance with gene detection, as shown in Fig. 4.

\section{Discussion}

Imipenem is stable to a lot of $\beta$-lactamase or super spectrum $\beta$-lactamase and it has strong affinity in penicillin binding proteins. Additionally, it has no cross resistance with the third generation of cephalosporin, with powerful bacterial activities. Therefore, it has become a common antibiotic in treating gram-negative bacilli, especially Pseudomonas aeruginosa infection (6). It has been widely used clinically, but in the presence of other antibiotics the detection rate of drug resistance also gradually increased (7). This study has concluded that MBL positive rate was $40 \%$. The MIC value of IRPA was significantly higher than quality control bacteria and control bacteria. In total, 35 cases were screened out with IMP-1 positive, 20 with VIM-1 positive, 16 with SPM positive, 5 with 2 positive genes and 4 with 3 positive genes.

The structural gene OprD of coding OprD2 protein was located in the chromosome of bacteria. The changes of encoding gene sequences may cause OprD2 protein expression reduction or even missing and result in the decrease of OprE protein expression volume. When OprD2 was missing or its expression was decreased, it lead to permeability changes of bacterial outer membrane and then blocking the carbapenems entering into the bacteria, showing carbapenems resistance clinically (8). Additionally, OprD2 protein is able to form a specific locus by binding bacteria and carbapenems. It is one and only porin that allows antibiotics go through smoothly in current studies, especially for Pseudomonas aeruginosa (9). Therefore, loss or reduction in OMP OprD2 expression is an important mechanism leading to Pseudomonas aeruginosa resistance to carbapenem antibiotics. The present study suggests that if the rate of missing OprD2 proteins is $75 \%$, the rate of missing MBL positive strains could reach up to $90 \%$. The results of protein electrophoresis and gene sequencing were the same. Due to different regions and antibiotic habits, the detection rate of missing OprD2 protein can vary (10). However, there was still OprD2 protein expression positivity reported in MBL-positive strains, which indicated that missing 
OMP OprD2 was a major mechanism that causes IRPA, but it was not the single one. Some studies reported that pure OprD2 gene missing only has low level of IMP resistance while OprD2 missing can produce significant resistance to carbapenems antibiotics only if it acts together with other factors, such as producing chromosome-mediated OprD2 enzyme (11).

Some studies have suggested that the active efflux pump may play an important role in resistant Pseudomonas aeruginosa (12). It can positively expel antibiotics out of the bacteria. If the expression quantity of efflux pump increases, the antibiotic output also goes up resulting in bacteria resistant to this antibiotic. The MexAB-OprM efflux system is the most common and most important active efflux pump in Pseudomonas aeruginosa. Its regulatory gene MexAB-OprM operon may be affected by upstream repressor gene MexR coded MexR protein. Its substrate is relatively extensive mainly including $\beta$-lactamase inhibitor, $\beta$-lactam, tetracyclines, chloramphenicol, quinolones, novobiocin, macrolides, sulfonamides and carbapenems. It usually causes multidrug resistance (13).

In conclusion, missing OMP OprD2 and MBL phenotype positivity may be the important mechanisms for IRPA.

\section{Acknowledgements}

This study was supported by the National Natural Science Foundation of China (no. 81260663) and the Health Department Fund Project of Guangxi Province (no. z2013045).

\section{References}

1. Strateva T and Yordanov D: Pseudomonas aeruginosa - a phenomenon of bacterial resistance. J Med Microbiol 58: $1133-1148,2009$.

2. Aktaş Z, Satana D, Kayacan C, Can B, Gönüllü N and Küçükbasmacı O: Antibiotic susceptibility rates and beta-lactam resistance mechanisms of Pseudomonas aeruginosa strains. Mikrobiyol Bul 46: 386-397, 2012 (In Turkish).
3. Bahar MA, Jamali S and Samadikuchaksaraei A: Imipenemresistant Pseudomonas aeruginosa strains carry metallobeta-lactamase gene bla(VIM) in a level I Iranian burn hospital. Burns 36: 826-830, 2010.

4. Riera E, Cabot G, Mulet X, García-Castillo M, del Campo R, Juan C, Cantón R and Oliver A: Pseudomonas aeruginosa carbapenem resistance mechanisms in Spain: Impact on the activity of imipenem, meropenem and doripenem. J Antimicrob Chemother 66: 2022-2027, 2011.

5. Epp SF, Köhler T, Plésiat P, Michéa-Hamzehpour M, Frey J and Pechère JC: C-terminal region of Pseudomonas aeruginosa outer membrane porin OprD modulates susceptibility to meropenem. Antimicrob Agents Chemother 45: 1780-1787, 2001.

6. Clissold SP, Todd PA and Campoli-Richards DM: Imipenem/cilastatin. A review of its antibacterial activity, pharmacokinetic properties and therapeutic efficacy. Drugs 33: 183-241, 1987.

7. Davodian E, Sadeghifard N, Ghasemian A and Noorbakhsh S: Presence of blaPER-1 and blaVEB-1 beta-lactamase genes among isolates of Pseudomonas aeruginosa from South West of Iran. J Epidemiol Glob Health: Mar 1, 2016 (Epub ahead of print).

8. Li H, Luo YF, Williams BJ, Blackwell TS and Xie CM: Structure and function of OprD protein in Pseudomonas aeruginosa: From antibiotic resistance to novel therapies. Int J Med Microbiol 302: 63-68, 2012.

9. Shen J, Pan Y and Fang Y: Role of the outer membrane protein OprD2 in carbapenem-resistance mechanisms of Pseudomonas aeruginosa. PLoS One 10: e0139995, 2015.

10. Shen JL and Fang YP: Detection of drug-resistance mechanism of Pseudomonas aeruginosa developing from a sensitive strain to a persister during carbapenem treatment. Genet Mol Res 14: 6723-6732, 2015.

11. El-Mahdy TS: Expression of ampC, oprD, and mexA, outer membrane protein analysis and carbapenemases in multidrug resistant clinical isolates of Pseudomonas aeruginosa from Egypt. J Chemother 26: 379-381, 2014.

12. Chuanchuen R, Wannaprasat W and Schweizer HP: Functional characterization of MexXY and OpmG in aminoglycoside efflux in Pseudomonas aeruginosa. Southeast Asian J Trop Med Public Health 39: 115-122, 2008.

13. Choudhury D, Ghosh A, Dhar Chanda D, Das Talukdar A, Dutta Choudhury M, Paul D, Maurya AP, Chakravorty A and Bhattacharjee A: Premature termination of MexR leads to overexpression of MexAB-OprM efflux pump in Pseudomonas aeruginosa in a tertiary referral hospital in India. PLoS One 11: e0149156, 2016. 